\title{
An equatorial oscillation in Saturn's middle atmosphere
}

\author{
T. Fouchet $^{1}$, S. Guerlet ${ }^{1}$, D. F. Strobel ${ }^{2}$, A. A. Simon-Miller ${ }^{3}$, B. Bézard ${ }^{1}$ \& F. M. Flasar ${ }^{3}$
}

The middle atmospheres of planets are driven by a combination of radiative heating and cooling, mean meridional motions, and vertically propagating waves (which originate in the deep troposphere). It is very difficult to model these effects and, therefore, observations are essential to advancing our understanding of atmospheres. The equatorial stratospheres of Earth and Jupiter oscillate quasi-periodically on timescales of about two and four years, respectively, driven by wave-induced momentum transport $^{1-5}$. On Venus and Titan, waves originating from surfaceatmosphere interaction and inertial instability are thought to drive the atmosphere to rotate more rapidly than the surface (superrotation). However, the relevant wave modes have not yet been precisely identified. Here we report infrared observations showing that Saturn has an equatorial oscillation like those found on Earth and Jupiter, as well as a mid-latitude subsidence that may be associated with the equatorial motion. The latitudinal extent of Saturn's oscillation shows that it obeys the same basic physics as do those on Earth and Jupiter. Future highly resolved observations of the temperature profile together with modelling of these three different atmospheres will allow us determine the wave mode, the wavelength and the wave amplitude that lead to middle atmosphere oscillation.

The stratosphere of Saturn is a region where temperatures increase with altitude, implying a large static stability and important wave activity. The upper stratosphere has a rich hydrocarbon chemistry that is initiated by methane photolysis and produces several species, from acetylene to benzene, which affect temperatures through their radiative properties. Unfortunately, observations performed before the arrival of the NASA Cassini spacecraft were generally limited in vertical coverage to the lower stratosphere (pressures greater than $1 \mathrm{hPa}$ ), where the dynamical and chemical timescales were longer than or comparable to a saturnian year ${ }^{6-9}$. Observations were also restricted to the summer hemisphere because the winter hemisphere was always hidden behind the optically thick rings. Cassini now gives us access to the full stratospheric range over the whole planet, in particular the upper stratosphere, where the seasonal forcing triggers rapid observable changes.

We acquired limb thermal spectra of Saturn for 2005-2006 using the Composite Infrared Spectrometer (CIRS) aboard Cassini. Limb viewing provides us with a vertical resolution of $\sim 1.5$ times the scale height $(\sim 75 \mathrm{~km})$ and probes higher altitudes than nadir sounding ${ }^{10}$. Figure la displays the pressure-latitude cross-section of the zonalmean temperature inverted from these CIRS limb spectra. Of particular note is the sharp difference in vertical structure between the northern and southern mid-latitudes and the equatorial region. At extratropical latitudes, the temperature steadily increases with altitude, whereas in the equatorial region it oscillates, with maximum amplitude centred on the equator. Although our temperature retrievals agree with previous measurements ${ }^{11,12}$ wherever the latter are available, only the vertical resolution and the horizontal coverage on both sides of the equator provided by CIRS limb observations allow us to detect and map the equatorial vertical oscillation. Using the thermal-wind equation (see Supplementary Information), we derived a zonal thermal-wind pressure-latitude cross-section for the stratosphere (Fig. 1b). The vertical structure of the zonal thermal wind at the equator clearly shows alternate bands of eastward and westward vertical shear from the $0.2-\mathrm{hPa}$ altitude down to the $5-\mathrm{hPa}$ altitude.

This temperature and zonal wind structure resembles those of Earth's quasi-biennial oscillation (QBO) and Jupiter's quasiquadrennial oscillation (QQO), in which temperature anomalies and eastward/westward winds alternate in altitude ${ }^{1-5}$. As the QBO and QQO vertical structures of alternating temperature anomalies and winds propagate down, the equatorial wind regime at a given height cyclically changes from easterly to westerly. On Earth, the alternating wind regimes repeat at intervals that vary from 22 to 34 months, with an average period of about 28 months $^{1,2}$. On Jupiter, the equatorial stratospheric temperature exhibits a 4.4 -year period and the equatorial zonal winds in the upper troposphere oscillate with a 4.5 -year period ${ }^{3,13,14}$. Long-term ground-based monitoring reveals a period of $14.7 \pm 0.9$ terrestrial years on Saturn ${ }^{15}$. The observational similarities between Saturn's oscillation and the QBO and QQO are the strong equatorial confinement of temperature minima and maxima and associated shear layers, a stronger eastward than westward shear layer, and the bounding of the equatorial oscillation at latitudes $15-20^{\circ}$ north and south. Temperatures near these latitudes are relatively high when equatorial temperatures are relatively low, and vice versa.

On Earth and Jupiter, the quasi-periodic oscillations are triggered by the interaction between upwardly propagating waves and the mean zonal flow ${ }^{2,46-20}$. Planetary-scale Kelvin waves contribute to eastward stresses, whereas mixed Rossby-gravity waves contribute to westward stresses. Over time, the zonal-mean wind anomalies descend and are damped by viscous processes at the tropopause, leading to the observed quasi-periodic cycle. Observations of Saturn (either cloud tracking or thermal measurements ${ }^{21-24}$ ) show abundant evidence for the presence of waves of sizes ranging from planetary scale to mesoscale. These waves may be generated by the deep convection that carries the internal heat flux and waves generated by shear instabilities. Hence, Saturn exhibits conditions similar to the terrestrial and jovian environments that are favourable to the establishment of an equatorial oscillation driven by vertically propagating waves. A simple model that considers the equations for the evolution of a longitudinally symmetric atmosphere subject to a mechanical forcing shows that the equatorial oscillation should be confined within a distance $L$ of the equator (see Supplementary 
Information). A scale analysis of these equations yields the relation $L<(a D N /(2 \Omega))^{1 / 2}(\omega / \alpha)^{1 / 4}$, where $a$ is Saturn's radius, $D$ is a height scale, $N$ is the buoyancy frequency, $\Omega$ is Saturn's spin rate, $\alpha$ is a constant radiative timescale and $\omega$ is the mechanical forcing frequency. This scale analysis for Saturn yields $L=7,400 \mathrm{~km}$ (seven degrees of latitude), in satisfactory agreement with our observations.

At extratropical latitudes, the response of a rapidly rotating atmosphere to the forcing due to planetary-scale waves is quite distinct from that in the tropics: the forcing is balanced by the Coriolis force
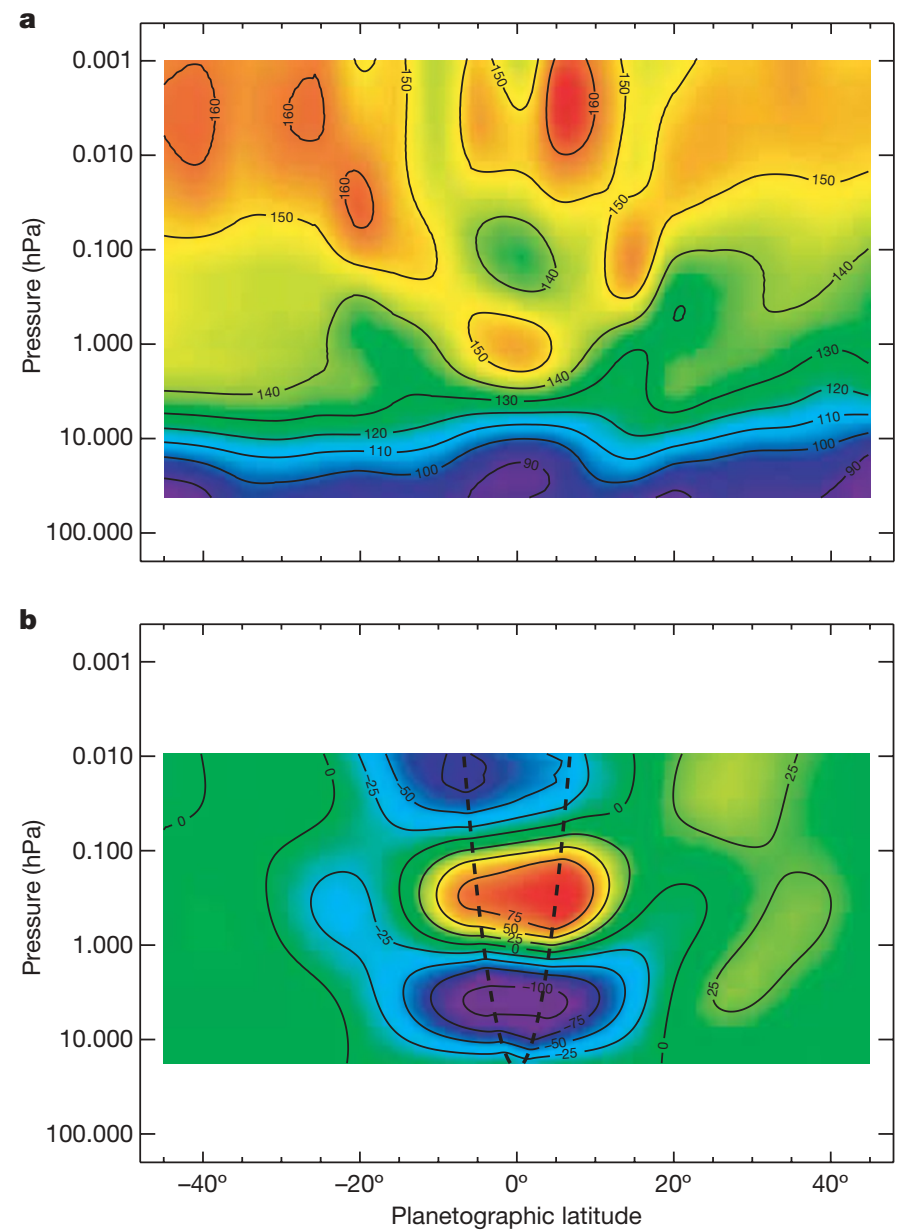

Figure 1 | Saturn's equatorial oscillation. a, This temperature map was retrieved using the hydrogen collision-induced continuum $\left(600 \mathrm{~cm}^{-1}\right)$ between the 20 and $2 \mathrm{hPa}$ pressure levels, and the $v_{4}$-band of methane $\left(1,300 \mathrm{~cm}^{-1}\right)$ between the 3 and $0.003 \mathrm{hPa}$ pressure levels (see Supplementary Information). The contours indicate temperature in Kelvin. The CIRS observed the planet's limb each $5^{\circ}$ of latitude between $45^{\circ} \mathrm{S}$ and $45^{\circ} \mathrm{N}$ (here and in $\mathrm{b}$, negative latitudes lie in the southern hemisphere). The ten detectors were aligned vertically, perpendicular to the planet's limb. Each detector independently probed a given altitude with a vertical resolution of $50-100 \mathrm{~km}$. The temperatures presented in this figure were retrieved from $15.5-\mathrm{cm}^{-1}$ spectra. Vertical profiles of atmospheric temperature were retrieved by means of a constrained inversion with strong low-pass filtering at each limb position. The number of independent longitudes sampled for a given latitude varies between two and four. At the equator the temperature near $10 \mathrm{hPa}$ is $17 \mathrm{~K}$ colder than it is at $20^{\circ} \mathrm{S}$ and $20^{\circ} \mathrm{N}$, whereas the equatorial temperature is $20 \mathrm{~K}$ warmer near $1 \mathrm{hPa}$; near $0.1 \mathrm{hPa}$ the equator is again $20 \mathrm{~K}$ colder, and at $0.01 \mathrm{hPa}$ the equatorial region is $10 \mathrm{~K}$ warmer than it is at adjacent latitudes. $\mathbf{b}$, This map presents the thermal wind field obtained by upward integration of the thermal wind equation on cylinders (see Supplementary Information). The contours indicate measured wind speed minus wind speed at $20 \mathrm{hPa}$, in metres per second. The dashed parabola delineates the exclusion zone within which the winds cannot be calculated from the thermal wind equation, but are instead interpolated. The error in temperature due to instrument noise is $2 \mathrm{~K}$, and this propagates to an error of $5 /$ (sine of latitude) $\mathrm{m} \mathrm{s}^{-1}$ per scale height in the zonal winds. produced by a mean meridional circulation. Indeed, at mid-latitudes, the stratospheric thermal profile that we retrieved differs completely from that in the equatorial region, in that it steadily increases with altitude. Surprisingly, the temperature difference between $45^{\circ} \mathrm{S}$ and $45^{\circ} \mathrm{N}$ at pressures between 10 and $1 \mathrm{hPa}$ is larger than that measured for pressures between 0.1 and $0.01 \mathrm{hPa}$. At $1-10 \mathrm{hPa}$ the temperature difference peaks at $15 \mathrm{~K}$, whereas at $0.01-0.1 \mathrm{hPa}$ it reaches only 6-7 K. The season observed on Saturn was southern midsummer (the solstice took place in October 2002). The observed temperature gradient between the southern and northern hemispheres should be close to its greatest seasonal amplitude ${ }^{8}$. Because the thermal inertia increases with depth, owing to larger optical depths, the latitudinal gradient was expected to be higher in the upper stratosphere than it is in the lower stratosphere.

To further explore this issue, we calculated the radiative cooling rates using the hydrocarbon vertical profiles inverted from CIRS limb data (Fig. 2). At $0.01 \mathrm{hPa}$ we obtained a similar cooling rate of $\sim 0.2 \mathrm{~K}$ per day for latitudes $30^{\circ} \mathrm{S}$ and $30^{\circ} \mathrm{N}$ (Fig. 3). This similarity in upper stratosphere cooling rates between the summer and winter hemispheres is in sharp contrast with the differences in the daily mean insolation, which combines the effects of Saturn's obliquity and screening by the thick rings. At the time of our observations, the ratio of the solar insolation at $30^{\circ} \mathrm{S}$ to that at $30^{\circ} \mathrm{N}$ is greater than three (daily mean of $5 \mathrm{~W} \mathrm{~m}^{-2}$ in the southern hemisphere and $1.5 \mathrm{~W} \mathrm{~m}^{-2}$ in the northern hemisphere $\left.{ }^{6,9}\right)$. Hence, the observed state of the upper stratosphere requires either that the heating rate is dominated by a season-independent source or that heat is meridionally redistributed.

The meridional-vertical cross-section of the ethane abundance supports the hypothesis that heat is meridionally redistributed (Fig. 2). In the southern hemisphere, the observed equator-to-midlatitude abundance gradient agrees with the prediction of photochemical models that it should resemble the yearly average insolation rather than the current seasonal insolation'. However, in the northern hemisphere, the local maximum of the abundance in the upper atmosphere centred at $30^{\circ} \mathrm{N}$ matches neither the yearly average insolation nor the current insolation. This local maximum constitutes a second strong piece of evidence for transport of hydrocarbonrich air masses from the southern upper stratosphere to the northern hemisphere. We estimated the vertical velocity needed to explain the

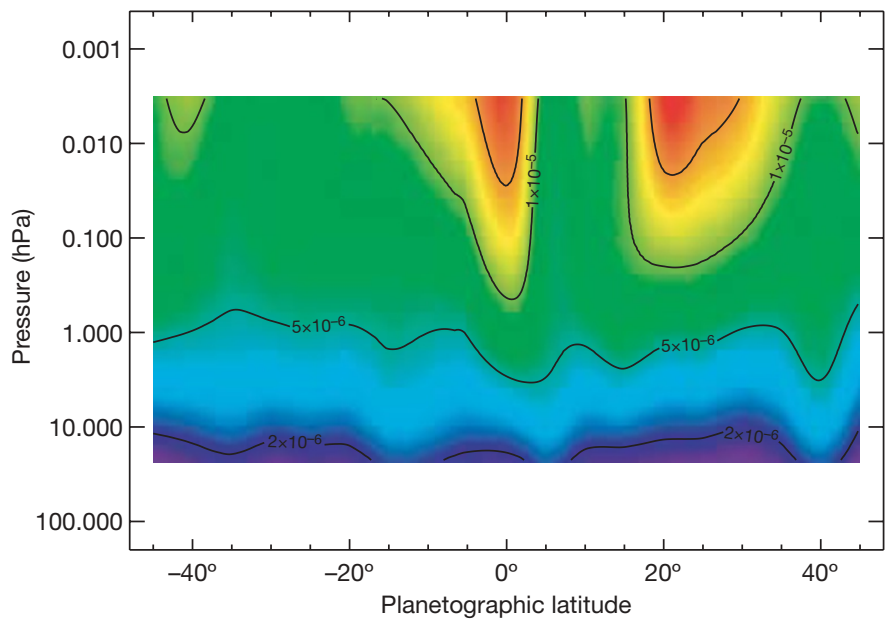

Figure 2 | Vertical-zonal map of ethane abundance. This pressure-latitude cross-section of the ethane volume mixing ratio (indicated by the contours) is inverted by fitting the $v_{9}$-band of ethane $\left(820 \mathrm{~cm}^{-1}\right)$ observed by the CIRS between 1 and $0.01 \mathrm{hPa}$. The temperature field used in the calculation is presented in Fig. 1. CIRS spectra measuring the temperature and the composition were obtained simultaneously at the same vertical and spectral resolution. Vertical profiles of the ethane volume mixing ratio were retrieved by means of a constrained inversion with strong low-pass filtering (see Supplementary Information). 


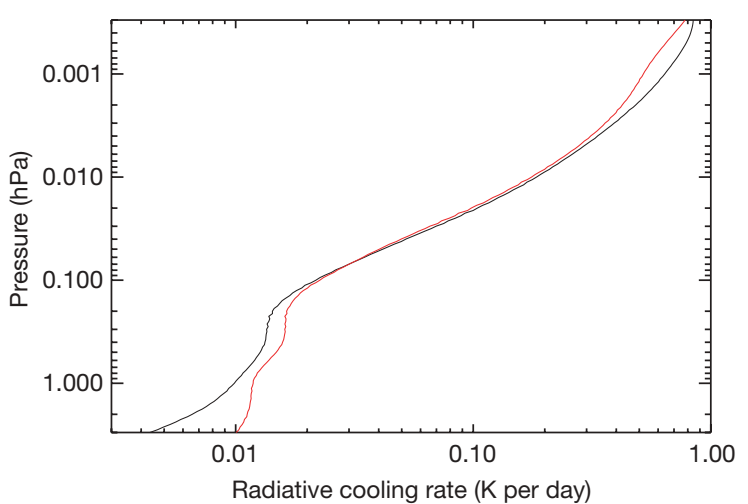

Figure 3 | Radiative cooling rates in the northern and southern hemispheres. The radiative cooling rates were calculated at $30^{\circ} \mathrm{N}$ (black) and $30^{\circ} \mathrm{S}$ (red) between 2 and $0.003 \mathrm{hPa}$, by differentiating the net thermal flux integrated over the $v_{4}$-band of methane, the $v_{9}$-band of ethane and the $v_{5}$-band of acetylene. The hydrocarbon abundance was set to the retrieved value presented in Fig. 2.

observed temperature, assuming adiabatic heating $\left(N^{2} H R^{-1} w=J / c_{p}\right.$, where $H$ is the atmospheric scale height, $w$ is the vertical wind speed, $J$ is the heating rate, $R$ is the gas constant and $c$ is the specific heat capacity at constant pressure). We did not calculate the solar heating rate, but we assumed that the atmosphere is globally in radiative balance, that is, that the total radiative cooling at $30^{\circ}$ south and north is equal to the total solar heating at these two latitudes. With a heating rate three times larger at $30^{\circ} \mathrm{S}$ than it is at $30^{\circ} \mathrm{N}$, this gives heating rates of 0.30 and $0.10 \mathrm{~K}$ per day, respectively, leading to a vertical velocity of $1.5 \mathrm{~mm} \mathrm{~s}^{-1}$.

This extratropical meridional circulation might be influenced by the equatorial oscillation. On Earth, the QBO does modulate the mean meridional circulation. Equatorial temperature minima are associated with local ascent and, by continuity, descent at extratropical latitudes. One interesting aspect of the global QBO is that the extratropical temperature anomalies are seasonally synchronized, occurring primarily during winter in each hemisphere. The reason for this is not fully known, but one possibility is that during northern winter the meridional flow from south to north, with subsidence in the northern hemisphere, will be reinforced by the QBO-induced sinking motion at extratropical latitudes. On Saturn, the latitude$15^{\circ}$ temperature anomalies at the $0.1-\mathrm{Pa}$ and $20-\mathrm{Pa}$ pressure levels are indeed warmer in the northern hemisphere than they are in the southern hemisphere. This seasonal asymmetry is another strong similarity in the behaviours of the stratospheres of Saturn and Earth.

\section{Received 10 December 2007; accepted 10 March 2008.}

1. Andrews, D. G., Holton, J. R. \& Leovy, C. B. Middle Atmosphere Dynamics (Academic, New York, 1987).

2. Baldwin, M. P. et al. The quasi-biennial oscillation. Rev. Geophys. 39, 179-230 (2001).
3. Leovy, C. B., Friedson, A. J. \& Orton, G. S. The quasiquadrennial oscillation of Jupiter's equatorial stratosphere. Nature 354, 380-382 (1991).

4. Friedson, A. J. New observations and modelling of a QBO-like oscillation in Jupiter's stratosphere. Icarus 137, 34-55 (1999).

5. Flasar, F. M. et al. An intense stratospheric jet on Jupiter. Nature 427, 132-135 (2004)

6. Barnet, C. D., Beebe, R. F. \& Conrath, B. J. A seasonal radiative-dynamic model of Saturn's troposphere. Icarus 98, 94-107 (1992).

7. Bézard, B. \& Gautier, D. A seasonal climate model of the atmospheres of the giant planets at the Voyager encounter time. I. Saturn's stratosphere. Icarus 60, 296-310 (1985).

8. Conrath, B. J., Gierasch, P. J. \& Leroy, S. S. Temperature and circulation in the stratosphere of the outer planets. Icarus 83, 255-281 (1990).

9. Moses, J. I. \& Greathouse, T. K. Latitudinal and seasonal models of stratospheric photochemistry on Saturn: Comparision with infrared data from IRTF/TEXES. J. Geophys. Res. 110, 09007 (2005).

10. Flasar, F. M. et al. Exploring the Saturn system in the thermal infrared: The Composite Infrared Spectrometer. Space Sci. Rev. 115, 169-297 (2004).

11. Flasar, F. M. et al. Temperatures, winds, and composition in the Saturnian system. Science 307, 1247-1251 (2005).

12. Greathouse, T. K. et al. Meridional variations of temperature, $\mathrm{C}_{2} \mathrm{H}_{2}$ and $\mathrm{C}_{2} \mathrm{H}_{6}$ abundances in Saturn's stratosphere at southern summer solstice. Icarus 177, 18-31 (2005).

13. Orton, G. S. et al. Thermal maps of Jupiter: Spatial organization and time dependence of stratospheric temperatures, 1980 to 1990 . Science 252, 537-542 (1991).

14. Simon-Miller, A. A., Poston, B. W., Orton, G. S. \& Fisher, B. Wind variations in Jupiter's equatorial atmosphere: A QQO counterpart? Icarus 186, 192-203 (2007).

15. Orton, G. S. et al. Semi-annual oscillations in Saturn's low-latitude stratospheric temperatures. Nature doi:10.1038/nature06897 (this issue).

16. Lindzen, R. S. \& Holton, J. R. A theory of the quasi-biennial oscillation. J. Atmos. Sci. 25, 1095-1107 (1968).

17. Holton, J. R. \& Lindzen, R. S. An updated theory for the quasi-biennial cycle of the tropical troposphere. J. Atmos. Sci. 29, 1076-1080 (1972).

18. Gray, L. J. \& Pyle, J. A. A two-dimensional model of the quasi-biennial oscillation in ozone. J. Atmos. Sci. 46, 203-220 (1989).

19. Dunkerton, T. J. Nonlinear propagation of zonal winds in an atmosphere with Newtonian cooling and equatorial wavedriving. J. Atmos. Sci. 48, 236-263 (1991).

20. Li, X. \& Read, P. L. A mechanical model of the quasi-quadrennial oscillation in Jupiter's stratosphere. Planet. Space Sci. 48, 637-669 (2000).

21. Beebe, R. F., Barnet, C., Sada, P. V. \& Murrell, A. S. The onset and growth of the 1990 equatorial disturbance on Saturn. Icarus 95, 163-172 (1992).

22. Sanchez-Lavega, A. Observations of Saturn's Ribbon Wave 14 years after Its discovery. Icarus 158, 272-275 (2002).

23. Achterberg, R. K. \& Flasar, F. M. Planetary-scale thermal waves in Saturn's upper troposphere. Icarus 119, 350-369 (1996).

24. Schinder, P. J. et al. Vertically propagating waves in the upper atmosphere of Saturn from Cassini radio occultations. (AGU Fall Meeting Abstract No. P23D06, 2005).

Supplementary Information is linked to the online version of the paper at www.nature.com/nature.

Author Contributions T.F. and S.G. inverted the temperature and composition from CIRS limb observations. T.F., F.M.F. and D.F.S. integrated the thermal wind equation. T.F., S.G. and B.B. calculated the radiative cooling rates. A.A.S.-M. designed the observations.

Author Information Reprints and permissions information is available at www.nature.com/reprints. Correspondence and requests for materials should be addressed to T.F. (thierry.fouchet@obspm.fr). 